\title{
Propuesta de un proceso educativo de habilidades del pensamiento como estrategias de aprendizaje en las organizaciones
}

\author{
Luz María Montoya Pérez *
}

\section{Resumen}

El presente trabajo busca contribuir modestamente al conocimiento del aprendizaje individual y grupal dentro de las organizaciones; para ello, se aplica un proceso educativo enfocado a reafirmar habilidades de pensamiento a partir del diagnóstico personal y organizacional del desempeño en el trabajo, incluyendo aspectos de tipo valorativo y de relaciones interpersonales.

Como parte de la investigación, se diseñó un proceso educativo compuesto por sesiones dirigidas y otras autodirigidas, una serie de ejercicios escritos que resolvieron los participantes para fortalecer cinco habilidades de pensamiento: análisis, relación, comparación, aplicación y deducción. Esto favoreció algunas operaciones cognitivas, principalmente la reflexión, la proposición, la descripción y la discriminación. Se manejaron dos grupos de participantes con nivel de educación media y profesionistas, en su gran mayoría ingenieros.

Se partió de tres vertientes: la psicología cognitiva en lo referente a las habilidades de pensamiento y los hábitos para pensar; desde la administración se consideraron las propuestas de algunos autores relevantes en el campo del aprendizaje organizacional; finalmente, la tercera a partir de la psicología educativa y toma como base las teorías del aprendizaje de corte constructivista.

Palabras clave: aprendizaje en las organizaciones, capacitación, habilidades de pensamiento, estrategias de aprendizaje.

\footnotetext{
*Profesora de la División de Estudios de Posgrado de la Facultad de Contaduría y Administración, UNAM. Correo electrónico: luzmontoya@att.net.mx
} 


\section{Encuadre de las habilidades del pensamiento}

T as habilidades del pensamiento están directamente relacionadas con la cogniLción, que se refiere a conocer, recoger, organizar y utilizar el conocimiento. La cognición se relaciona con muchos otros procesos, prácticamente con todos aquellos que involucren percepción, memoria, aprendizaje; esto implica que todas las actividades derivadas del pensamiento tienen componentes cognitivos.

En ese sentido, pensar se ha asumido generalmente como un proceso cognitivo, un acto mental a través del cual es adquirido el conocimiento. Sin embargo, la cognición incluye diferentes formas de conocer algo, incluyendo la percepción, el razonamiento y la intuición; de éstas, el razonamiento se considera como la habilidad más importante del pensamiento.

El aprendizaje es una consecuencia del pensamiento porque de los procesos de éste se derivan: ideas, conocimientos, conclusiones y argumentos; en un nivel más elevado se encuentran: juicios, solución de problemas y análisis crítico.

Algunas aproximaciones a los procesos de pensamiento son propuestas por diferentes autores, entre los que destacan Labarrere (1994), quien considera que el pensamiento es un proceso de búsqueda, de descubrimiento, de investigación constante, que se manifiesta a través de la elaboración de hipótesis, razonamientos y emisión de juicios. ${ }^{1}$

Bereiter y Scardamalia (1985) propusieron que la escritura era una oportunidad para pensar; además, señalaron a ésta como un reflejo y uno de los principales vehículos del pensamiento, pues exige que quien escribe razone, por lo que es considerada como estrategia cognitiva. ${ }^{2}$

Aunque pensar es una actividad normal que ocurre sin necesidad de entrenamiento, es importante incrementar la habilidad para aplicar las diferentes operaciones

\footnotetext{
${ }^{1}$ Labarrere, Arthur, Pensamiento, análisis y autorregulación en la actividad cognoscitiva de los alumnos, p. 132.

${ }^{2}$ Bereiter, C. y M. Scardamalia, "Cognitive coping strategies and the problem of inert-knowledege", en Chipman, S., J. Segal y R. Glaser (Comps.), Thinking and learning skills. Research and open questions, vol. 2 , p. 45 .
} 
cognitivas como observar, opinar, inferir, evaluar, entre otras, a través de su práctica consciente; en este sentido, se busca ayudar a las personas a mejorar sus rendimientos cognitivos.

Desde la perspectiva del individuo, el conocimiento se define como el conjunto de representaciones de la realidad que lo reproduce o lo construye y que se encuentra almacenado en su memoria. Así, para Derry y Murphy (1986) las estrategias cognitivas son el conjunto de procedimientos o procesos mentales que utiliza una persona en una situación particular de aprendizaje para facilitar la adquisición de conocimientos. $^{3}$

Las habilidades del pensamiento no se pueden identificar en forma completamente pura, sino que cada una de ellas requiere de otras; por ejemplo, para sintetizar es necesario identificar las ideas principales (habilidad de análisis) o para hacer una inferencia se requiere previamente comparar e identificar relaciones.

Por su parte, Marzano et al. (1988) han propuesto 21 operaciones cognitivas agrupadas en ocho habilidades de pensamiento básicas: ${ }^{4}$

\section{Habilidades de enfoque}

1. Definir problemas

2. Establecer metas

\section{Habilidades para recopilar información}

3. Observar

4. Formular preguntas

\section{Habilidades de memoria}

5. Almacenar

6. Recordar

\footnotetext{
${ }^{3}$ Derry, S. y D. Murphy, "Designing systems that train learning ability: From theory to practice", en Review of Educational Research, pp. 1-39.

${ }^{4}$ Marzano, Robert et al., Dimensions of thinking. A framework for curriculum and instruction.
} 


\section{Habilidades de organización}

7. Comparar

8. Clasificar

9. Ordenar

10. Representar

\section{Habilidades de análisis}

11. Identificar atributos y componentes

12. Identificar relaciones y patrones

13. Identificar ideas principales

14. Identificar errores

\section{Habilidades de construcción}
15. Inferir
16. Predecir
17. Elaborar

\section{Habilidades de integración}

18. Resumir

19. Reestructurar

\section{Habilidades de evaluación}

20. Establecer criterios

21. Verificar

Asimismo, otra habilidad cognitiva fundamental es la metacognición, que implica el incremento de la conciencia de los procesos de pensamiento por sí mismos, así como conocer los procesos individuales como pensador y ejecutor; es el conocimiento del conocimiento, incluyendo el conocimiento de las capacidades y limitaciones de los procesos del pensamiento. Como aprendices, se requiere ejercitar los diferentes procesos de pensamiento para entenderlos y aplicarlos mejor. A este respecto, Presseisen (2001) propone el siguiente modelo de habilidades metacognitivas: ${ }^{5}$

5 Presseisen, G., en Costa, A. (2001), Developing Minds. Association for Supervision and Curriculo Development, p. 157. 


\section{Me t a c o g n i c i ó $n$}

Monitorear el desempeño de las tareas

Mantener dirección, secuencia

Detectar y corregir errores

Incrementar el trabajo

Producto: mayor exactitud en el desempeño de la tarea
Seleccionar y entender una estrategia apropiada

Focalizar la atención sobre lo que se necesita

Relacionar lo que se conoce con el material por ser aprendido

Probar lo correcto de una estrategia

Producto: mayor habilidad para completar procesos de pensamiento

Para los fines de este trabajo se conformaron, con base en Marzano (1988) y Presseisen (2001), las siguientes cinco habilidades de pensamiento con sus operaciones cognoscitivas respectivas:

\section{Categoría I: análisis}

1. Descomponer en dos o más ideas

2. Identificar situaciones

3. Identificar ideas principales

4. Seleccionar entre un grupo de cosas

\section{Categoría II: comparar}

1. Establecer jerarquías valorativas

2. Identificar semejanzas

3. Identificar diferencias

4. Establecer jerarquías cuantitativas

\section{Categoría III: relacionar}

1. Establecer conexiones

2. Hacer analogías

3. Establecer dependencias: causales, tiempo, personas, procesos

4. Establecer relaciones condicionales 


\section{Categoría IV: aplicar}
1. Hacer transferencias de ideas
2. Proponer formas de utilizar algo
3. Dar ejemplos
4. Hacer evaluaciones

\section{Categoría V: deducir}

1. Sacar conclusiones

2. Hacer inferencias

3. Identificar consecuencias

4. Dar razones o argumentos

A continuación se plantea la relevancia del aprendizaje en las organizaciones desde la perspectiva de la administración; para ello se parte de los productos de las habilidades intelectuales como son la generación de ideas, propuestas, innovaciones, etc., en el desempeño del trabajo cotidiano.

\section{El aprendizaje para el desempeño en el trabajo. Enfoque de la administra- ción}

Los desafíos que se enfrentan en el trabajo tienen una connotación altamente compleja derivada de los cambios permanentes donde se encuentran inmersas las organizaciones, en especial en el contexto de la globalización, lo que exige de los individuos cambios en su interpretación del entorno del trabajo para de esta forma asimilar nuevas ideas, técnicas o prácticas. ${ }^{6}$

Desde esta perspectiva, para estar involucrado en el trabajo es necesario estarlo también en el aprendizaje, lo que puede apreciarse desde diferentes perspectivas: el trabajo puede presentar retos profesionales, así como retos intelectuales; también implica la posibilidad de adquirir nuevas experiencias en otros campos. De esta manera, el aprendizaje provee oportunidades para el cambio personal y el desarrollo profesional.

El aprendizaje dentro del trabajo se ha convertido en uno de los campos de desarrollo más importantes como área común de la administración y la educación.

${ }^{6}$ Boud, D. y J. Garrick, Understanding learning at work, p. 3. 
Ahora los empleados amplían sus capacidades educativas al aprender a través de su trabajo, ya que las oportunidades y los problemas laborales cotidianos les demandan nuevos conocimientos y desafíos. Así, las personas no sólo realizan sus responsabilidades eficientemente, sino que ayudan a transformar el entorno del trabajo donde están involucrados a través de crear mejores prácticas y formas de producción.

Las opciones educativas dentro de las empresas se pueden ubicar en tres planos: educación formal, educación no formal y educación informal. La educación formal se realiza en escuelas o instituciones educativas y conducen a la obtención de títulos o acreditaciones. La oferta de educación no formal en las empresas se da a través de cursos, talleres y seminarios que están enfocados a la adquisición de conocimientos específicos; en general sólo implican conocimiento de carácter interno. La educación informal se basa principalmente en la interacción con otras personas, con la tecnología y el medio ambiente en general; sus efectos son en general poco evidentes.

El resumen de estas opciones educativas en las empresas se puede apreciar en el siguiente cuadro adaptado de Gore:

\begin{tabular}{|c|c|c|c|}
\hline & FORMAL & NO FORMAL & INFORMAL \\
\hline $\begin{array}{l}\mathrm{F} \\
\mathrm{O} \\
\mathrm{R} \\
\mathrm{M} \\
\mathrm{A} \\
\mathrm{L}\end{array}$ & $\begin{array}{l}\text { Cursos o eventos } \\
\text { con reconocimiento } \\
\text { oficial o institucional } \\
\text { externo }\end{array}$ & $\begin{array}{l}\text { Autoformación, en pasantías, } \\
\text { participación en proyectos } \\
\text { conjuntos, reuniones para } \\
\text { solución de problemas, diversos } \\
\text { eventos o cursos de capacitación. }\end{array}$ & $\begin{array}{l}\text { Influencia de los } \\
\text { compañeros en los } \\
\text { programas de educación } \\
\text { formal. }\end{array}$ \\
\hline $\begin{array}{l}\mathrm{N} \\
\mathrm{O} \\
\mathrm{F} \\
\mathrm{O} \\
\mathrm{R} \\
\mathrm{M} \\
\mathrm{A} \\
\mathrm{L}\end{array}$ & $\begin{array}{l}\text { Acreditación interna } \\
\text { de cursos impartidos } \\
\text { por la empresa }\end{array}$ & $\begin{array}{l}\text { Especialización en el trabajo, } \\
\text { identificación de mejores } \\
\text { prácticas de trabajo, orientación } \\
\text { brindada por el jefe inmediato, } \\
\text { visitas de benchmarking }\end{array}$ & $\begin{array}{l}\text { Aprendizaje a través de la } \\
\text { participación, compartir } \\
\text { información } \\
\text { conocimientos, lecturas de } \\
\text { reflexión, manuales de } \\
\text { proveedores. }\end{array}$ \\
\hline $\begin{array}{l}\text { I } \\
\text { N } \\
\text { F } \\
\text { O } \\
\text { R } \\
\text { M } \\
\text { A } \\
\text { L }\end{array}$ & $\begin{array}{l}\text { Procedimientos, } \\
\text { juntas, } \\
\text { comunicación interna, } \\
\text { intranet. }\end{array}$ & $\begin{array}{l}\text { Revistas técnicas, internet, } \\
\text { libros diversos. }\end{array}$ & $\begin{array}{l}\text { Experiencias diarias, } \\
\text { aprender haciendo, } \\
\text { modelaje }\end{array}$ \\
\hline
\end{tabular}

Fuente: E. Gore, La educación en la empresa, Barcelona, Edit. Granica, 1998 
Por lo anterior, la empresa se considera como un ámbito importante de educación; de aquí la necesidad de que los individuos reflexionen e identifiquen posibilidades de aprendizaje formal, no formal e informal de acuerdo con sus necesidades inmediatas, sus planes a mediano y largo plazo, así como sus intereses.

Sin embargo, los enfoques tradicionales de formación dentro de las empresas no han dado suficiente importancia a la enseñanza de habilidades de pensamiento relacionadas con actividades de orden superior: razonamiento, pensamiento creativo y solución de problemas, ni siquiera para reforzar las habilidades cognitivas básicas como la diferenciación, la aplicación, la demostración, entre otras.

Asimismo, es necesario que los individuos aprendan a utilizar las oportunidades educativas brindadas por las organizaciones como una estrategia de crecimiento personal y profesional, lo que justifican Candy and Matthews (1998) señalando los siguientes roles del lugar de trabajo en el aprendizaje:

\section{El lugar de trabajo es:}

- un sitio para acreditar formalmente el aprendizaje

- un lugar para interacciones técnicas complejas y solución de problemas

- es un sitio para compartir y crear conocimiento

- es una parte de la sociedad del conocimiento

- es una entidad orgánica, capaz de aprender y adaptarse en su propio beneficio ${ }^{7}$

Por su parte, Argyris y Schön (1990) mencionan que el aprendizaje en las organizaciones implica la detección y corrección del error, así como tres niveles de aprendizaje: ciclo simple, doble ciclo y deutero aprendizaje (aprender a aprender), de acuerdo con el camino que se siga al detectar y corregir los errores. Estos autores proponen que el aprendizaje de circuito simple es el que coincide con las experiencias y los valores de la persona, lo que le permite responder de manera automática, como pueden ser las actividades que exigen seguir procedimientos muy establecidos. El aprendizaje de doble circuito es el que incluye la retroalimentación en cuanto a los errores, implica tanto cambio en las cosas que se asumen, como en las estrategias, las cuales pueden generar, como consecuencia, un cambio en los valo-

\footnotetext{
${ }^{7}$ Candy, P. y J. Matthews, en Understanding learning at work, p. 148.
} 
res. Estos cambios pueden resultar de un proceso de introspección por parte de los individuos. El deutero aprendizaje es el que no concuerda con ciertos elementos de la organización y requiere que se cambien esquemas mentales. ${ }^{8}$

\section{Influencia de la cultura organizativa en el aprendizaje}

La cultura organizativa es un patrón de supuestos básicos, creados por un grupo de personas, a partir de los cuales se comparten significados, mitos y creencias para enfrentar la incertidumbre, así como su necesidad de responder a las demandas externas manteniendo la coherencia interna. (Schein, 1999). ${ }^{9}$

Para Gore (1998), en una determinada cultura organizativa puede predominar alguna de las siguientes orientaciones: hacia los resultados, hacia el poder, hacia la gente, hacia los procedimientos; cada orientación podría favorecer determinados aprendizajes. A continuación se explica cada orientación. ${ }^{10}$

- En una cultura orientada hacia los resultados, se requiere aprender criterios de eficiencia, cumplir los requerimientos de evaluación de logros; se evalúan las ideas, las cosas y las personas por su posibilidad de conseguir los resultados.

- En una cultura dirigida hacia el poder, se aprende a conseguir influencia; así los logros, la autoridad y el éxito están determinados con relación a la estabilidad política de la empresa.

- En una cultura centrada en las personas, se otorga un gran valor a conservar las buenas relaciones, se desecha el conflicto y se busca mantener la armonía entre la gente.

- En las culturas basadas en los procedimientos, se otorga mucho más peso a las normas que rigen las acciones que al logro de las metas y que a la reflexión sobre cómo se realizan esas acciones.

\footnotetext{
${ }^{8}$ Argyris, C. y D. Schon, Organizational Learning: a theory of action perspective. Reading, p. 59.

${ }^{9}$ Schein, E. (1999), "On dialogue, culture, and organizational learning", en Organizational Dynamics, pp. 124-135.

${ }^{10}$ Gore, E., op. cit.
} 
En cualquiera de estas orientaciones, toda persona que tiene mando trabaja con ideas, cosas y personas. Se trabaja con ideas al planear, al proponer, al innovar, al fijar objetivos, al establecer prioridades, al interpretar las señales del entorno, al definir problemas. Se trabaja con objetos al organizar, al asignar recursos, al cuidar un presupuesto, al manejar tecnologías, al generar riqueza. Se trabaja con personas al dirigir, al formar equipos, al capacitar a los colaboradores, al controlar, al buscar persuadir.

De esta manera, la forma como influyen las culturas organizativas va más allá de los valores y actitudes, determinando los criterios cognitivos sobre los que se construye la realidad, por lo que condicionan el aprendizaje a partir del tratamiento de la información. Al construir un lenguaje, categorizar a la gente, las ideas y las cosas en función de su peculiar transformación de la realidad, las organizaciones condicionan procesos cognitivos básicos de sus miembros. En este sentido, el hecho de que condicione el pensamiento individual convierte a las organizaciones en paradigmas de nuestra sociedad. ${ }^{11}$

Sin embargo, existen bloqueos organizativos que dificultan trabajar con las ideas, las cosas y las personas, algunos son rutina excesiva, falta de información, crisis constantes, falta de compromiso, poca claridad en los proyectos, liderazgos erráticos, falta de motivación, escaso espíritu de equipo, alta rotación de personal, batallas internas entre áreas, falta de iniciativa, escasez de recursos, falta de capacitación, cultura de lo urgente contra lo importante. Adicionalmente, la forma en que el edificio está construido, la división del trabajo, las comunicaciones (quiénes hablan y de qué), las recompensas y las sanciones, las normas escritas y de otro tipo, todo confluye a condicionar el quehacer humano, a volverlo previsible en función de ciertos objetivos.

\section{Enfoque de la psicología educativa: la aproximación constructivista al apren- dizaje}

La participación de la psicología educativa en la investigación del aprendizaje se enfoca básicamente en varias direcciones. Una de ellas estudia los procesos intelectuales que afectan el aprendizaje — tanto en su adquisición como en su retención a largo plazo—, investiga la mejora de las capacidades para aprender y

11 Gore, D., op. cit. 
resolver problemas, estudia las características cognoscitivas — de personalidad y de aspectos sociales que afectan los resultados del aprendizaje - y determina las maneras más eficaces de organizar y presentar los materiales de aprendizaje.

Desde el constructivismo, el conocimiento es una construcción del individuo a partir de los esquemas que posee y que tomó como base para su interrelación con el medio ambiente que lo rodea. Entre los representantes más destacados del constructivismo se encuentra Ausubel (1973) con sus propuestas sobre el aprendizaje significativo desde una reestructuración activa de las percepciones, ideas, conceptos y esquemas que la persona posee en su estructura cognitiva. También plantea que el conocimiento posee significados lógicos y psicológicos, y que cada persona transforma el significado lógico en significado psicológico; este último no depende sólo de que la persona posea las aptitudes intelectuales y la base conceptual requerida, sino de su particular concepción sobre el tema o cuestión. De esta forma, cuando la persona aprende proposiciones lógicamente significativas, no aprende sólo su significado lógico, sino el que tienen para él. ${ }^{12}$

La relevancia de estos aspectos en el contexto laboral se refleja en la ejercitación para aplicar todos los contenidos teóricos a las situaciones cotidianas de su área de trabajo. En este sentido, la posibilidad de ampliar las habilidades intelectuales estudiadas se convierte en una forma de aprehensión en la medida que son herramientas que incrementan su potencial para aplicar su inteligencia.

Por otro lado, el aprendizaje puede verse inhibido por una estructura cognitiva inestable, ambigua y desorganizada, esto significa que intervienen las propiedades organizativas del conocimiento que posee la persona que aprende, como la simplificación, la inconsistencia, la dispersión, o bien aspectos subjetivos de idiosincrasia, o tendencias como la intolerancia a la ambigüedad. En este aspecto es importante fortalecer la estructura cognitiva a través del fortalecimiento de las habilidades de pensamiento con el fin de que el aprendizaje se realice con economía de tiempo y esfuerzo mediante procesos como la abstracción, generalización y diferenciación, entre otros.

En el contexto de las organizaciones, se busca que las personas tengan conciencia de que el aprendizaje se da de manera gradual y lo que se aprende o domina sirve

${ }^{12}$ Ausubel D., Novak J. y H. Hanesian, Psicología educativa. Un punto de vista cognoscitivo, p. 61. 
como base para los requerimientos posteriores; asimismo, que tengan claridad sobre los productos del aprendizaje, que pueden ser traducidos en mejores prácticas, mayor participación, seguridad en la resolución de problemas, búsqueda de causas de los problemas, entre otros.

Para los fines de esta investigación, se parte de una posición teórica de corte constructivista enmarcada desde la administración en aspectos de aprendizaje para el desempeño, a partir del planteamiento de situaciones relacionadas con el trabajo cotidiano, encaminadas al análisis de los procesos personales de pensamiento.

A partir de estas bases teóricas, el objetivo central de la investigación consistió en aplicar un proceso educativo para trabajadores de diferentes niveles jerárquicos orientado a fortalecer cinco habilidades de pensamiento que se consideran especialmente relevantes en el desempeño de las funciones administrativas y de interacción humana, como herramientas de aprendizaje en una organización.

El total de participantes en la investigación se integró por 37 personas: 13 con escolaridad media y 24 con licenciatura, en su gran mayoría ingenieros. Se conformaron dos grupos: uno de 19 personas, 12 con un nivel de licenciatura y 7 con educación media; el segundo formado por 18 personas, 12 con un nivel de licenciatura y 6 con educación media. En cada grupo participaron supervisores de diferentes áreas de la empresa relacionados con procesos productivos y de calidad, así como gerentes vinculados con áreas productivas, de ingeniería y de calidad. Se buscó equilibrar la escolaridad de los participantes a cada grupo para poder correlacionar esta variable con respecto a los resultados encontrados en cada una de las categorías estudiadas.

Cada una de las cinco categorías de pensamiento y la escolaridad de los participantes se manejaron como variables dependientes. Como variable independiente se manejó el proceso educativo, que contenía ejercicios estructurados y materiales impresos administrados en cuatro sesiones conducidas por la investigadora y cuatro sesiones en un esquema de autoaprendizaje. En las sesiones autodirigidas, los participantes revisaban los contenidos y ejercicios correspondientes, que resolvían sin recibir ayuda por parte de la investigadora ni de sus compañeros.

\section{Método}

Los instrumentos para la recolección de los datos se dividieron en instrumentos para la intervención, guía para la identificación de categorías y formatos para el registro de las respuestas. 
Intervención: Se diseñó un manual como instrumento para medir las variables dependientes, conformado por materiales impresos, de aproximadamente 12 páginas para cada sesión, donde se desarrollaron los diferentes temas con contenidos teóricos breves, ilustrados para apoyar la relación visual con los diferentes contenidos, con un rango de 10 a 15 ejercicios en promedio por cada sesión y con un promedio de dos a tres ejercicios para cada una de las cinco habilidades de pensamiento estudiadas. Algunos ejemplos de ejercicios son los siguientes:

Ejercicio 2.8 Aplicación. Escoge cinco verbos del lenguaje del pensamiento que más utilices y plantea un ejemplo de cada uno que apliques al realizar tu trabajo.

Ejercicio 2.10 Deducción. Piensa en algo que hayas aprendido recientemente y explica el proceso que pasaste para lograrlo.

Ejercicio 3.4 Relación. ¿Cómo puedes aprender a cuidar los costos en las actividades que realizas en tu puesto de trabajo?

Ejercicio 3.7 Análisis. ¿Cuáles son los requisitos de calidad que debes cumplir al realizar tu trabajo?

Ejercicio 5.2 Comparación. Escribe un ejemplo donde puedas comparar los tres puntos del modelo revisado al trasladarlo a una situación de motivación personal en el trabajo.

Tanto el entrenamiento dirigido como los contenidos se diseñaron para interesar a los participantes de manera paulatina por aprender a través de la identificación de posibilidades de ejercitar sus capacidades para pensar, así como identificar los beneficios que pueden obtener no sólo en el trabajo, sino en su crecimiento personal, familiar y social. La gran mayoría de ejercicios se relaciona con situaciones de su trabajo cotidiano. En este sentido se parte de la base que la identificación y empleo de habilidades cognitivas — como posibilidad para aprender- les brindará una plataforma para continuar aplicándolas como herramientas en sus actividades cotidianas, tanto en el trabajo como en su vida personal.

El manual se conformó con 85 ejercicios que los participantes resuelven a lo largo del entrenamiento y que además se orientan a cubrir ocho temáticas:

- Orientación del aprendizaje 
- Identificación del vocabulario del pensamiento

- Diagnóstico del desempeño

- Aprendizaje para el desempeño

- Motivación personal

- Análisis de problemas

- Innovación y creatividad

- Aprendizaje entre áreas

Se diseñó un instrumento como pre test para diagnosticar la situación de los participantes de ambos grupos antes de la intervención y como post test. Este instrumento se conformó de un total de 25 ejercicios: cinco para cada una de las variables o categorías de pensamiento estudiadas y se aplicó antes de iniciar el entrenamiento, y dos días después de haber finalizado.

Ambos grupos recibieron un entrenamiento combinado, cuatro sesiones de tipo presencial y cuatro bajo un esquema de autoaprendizaje, durante cuatro semanas. Se trabajó con cada grupo por separado, en ocho sesiones de dos horas de duración cada una, tanto para la modalidad dirigida como para la autodirigida. Las sesiones dirigidas se realizaron a través de un proceso educativo para fortalecer habilidades cognitivas, conducido por la investigadora en sesiones realizadas dos veces por semana. Para las sesiones autodirigidas se asignaron diferentes horarios de dos horas cada uno, con el objeto de que los participantes pudieran asistir a cubrir la sesión correspondiente en los horarios previamente establecidos.

\section{Justificación de las variables y dimensiones seleccionadas}

La selección de las cinco variables y dimensiones para la presente investigación tienen una fundamentación contextual en el análisis de las descripciones de puestos de los supervisores y de los gerentes de las dos empresas donde se realizó el estudio piloto y la segunda investigación. Con esta base, las justificaciones son las siguientes:

Se justifica la selección de la categoría Análisis porque las personas necesitan aplicar esta habilidad para implementar programas de calidad, en especial en relación con el aseguramiento de la calidad; al determinar estándares, al analizar las causas de las desviaciones; cuando dividen un problema complejo en etapas para su análisis; en cuestiones administrativas al revisar comunicados con el fin de captar su organización y estructura, así como identificar lo principal de lo secunda- 
rio; al dar soporte a una decisión; para determinar la secuencia de un plan a partir de establecer prioridades. Asimismo, analizar permite identificar la naturaleza de los hechos para discriminar si son juicios de valor o si tienen algún propósito.

La selección de la categoría Relación se justifica al encontrar las conexiones e interacciones entre elementos, por ejemplo, cuando implementan planes estratégicos que involucran a todas las áreas de la compañía; al distinguir entre las relaciones de causa y efecto y otras secuencias de relaciones; al controlar las variaciones en los procesos; al desarrollar proyectos, identificando etapas, secuencias, obstáculos, tiempos requeridos; al asociar hechos o experiencias anteriores con situaciones actuales y adaptarse a los nuevos requerimientos en su trabajo, como el cambio en la manufactura de productos. Con respecto al enfoque de resultados, a partir de plantear y cumplir los objetivos especificados; al determinar las condiciones y requerimientos para cumplir planes y metas; al considerar limitantes en tiempo y recursos; al identificar relaciones causales de tiempo, personas y procesos para elevar la eficiencia de su área de responsabilidad. También aplican esta habilidad al determinar necesidades diversas, tanto personales, grupales, de logística, de organización, de materiales y equipos, entre otras; asimismo, al identificar las consecuencias derivadas de llevar a la práctica determinados valores.

La selección de la categoría Comparación se justifica por las diversas aplicaciones en las funciones de su trabajo diario, entre ellas, al identificar patrones o tendencias en los procesos, al analizar problemas recurrentes, al identificar defectos en productos y al evaluar prácticas de trabajo y hábitos personales. En la toma de decisiones, por ejemplo, al seleccionar materiales, proveedores, etc; al identificar similitudes y diferencias en las partes y componentes de las máquinas y equipos; al establecer jerarquías cuantitativas en el control de procesos, especificaciones de calidad, llevar mediciones, registro de datos, cumplimiento de programas, control presupuestal, elaboración de informes y reportes; al buscar similitudes entre hechos o problemas; al realizar evaluaciones costo beneficio; al determinar brechas en el desempeño a partir de situaciones reales y deseadas; al identificar la influencia de diferentes valores en la realización de su trabajo; al revisar materias primas y productos terminados para determinar si cumplen con los estándares fijados con fines de control de calidad; al determinar las cualidades de los cambios ocurridos en diferentes situaciones.

La selección de la categoría Aplicación se justifica porque implica la comprensión de técnicas y métodos. Dentro de sus actividades cotidianas, los supervisores 
y gerentes utilizan esta categoría para determinar necesidades de capacitación: al resolver problemas diarios de diversos tipos; al atender imprevistos en el desempeño de sus funciones; al hacer propuestas en procesos, productos y empaques; al sugerir simplificaciones en procesos de producción o administrativos; al recomendar aditamentos en máquinas o mejores prácticas de trabajo para reducir tiempos; al transferir aspectos teóricos a la práctica, como aplicar principios de física en el mantenimiento de las máquinas o bien al aplicar el contrato de trabajo ante una situación de conflicto; al proponer acciones concretas para mejorar el desempeño, tanto individual como de sus colaboradores; al elaborar planes de trabajo; al adaptarse a cambios continuos; y al realizar autoevaluaciones relacionadas con operaciones cognitivas: cómo observo, cómo escucho, cómo justifico, cómo aclaro, etcétera.

La selección de la categoría Deducción se justifica debido a que los supervisores y gerentes requieren establecer relaciones entre hechos y llegar a conclusiones sobre problemas que se les presentan al evaluar los aspectos positivos y negativos de las situaciones, de donde se deriva la posibilidad de anticipar consecuencias para tomar decisiones; al analizar las causas de los problemas para dar una opinión o evaluación; al establecer analogías entre una secuencia de eventos, problemas o situaciones para hacer inferencias, como puede ser al identificar elementos comunes en una serie de problemas suscitados en un periodo determinado; al evaluar información o hechos para hacer propuestas diversas, así como al dar argumentos o razones para respaldarlas; al traducir aspectos abstractos como valores a situaciones tangibles de comportamiento; así como al anticipar problemas potenciales a partir de experiencias previas.

A partir de estas variables los ejercicios se conformaron para favorecer especialmente cuatro operaciones cognitivas fundamentales: reflexión, proposición, descripción y discriminación; la primera como base de la racionalidad, la segunda por su carácter práctico, la tercera y la cuarta por su relevancia para el análisis de problemas.

\section{Calificación de respuestas}

Se realizaron dos tipos de evaluaciones: cuantitativa y cualitativa. Para la evaluación cuantitativa se revisaron cuidadosamente todas las respuestas de los ejercicios de los 37 participantes en las cinco categorías, se vaciaron los datos en los formatos de codificación para asignar a cada respuesta valores de $0,0.5,1$, a partir de los siguientes criterios: 


\section{No acierto: valor 0}

Deja en blanco la respuesta.

La respuesta no tiene relación con lo que se pregunta.

Utiliza monosílabos.

Respuesta desarticulada.

Respuesta confusa, no hay claridad en lo que escribe.

Utiliza las mismas palabras de la pregunta para responder.

\section{Acierto parcial: valor 0.5}

Contesta de manera indirecta.

La respuesta es parcial, la pregunta se plantea para que conteste dos cosas y sólo responde una.

Responde correctamente sólo la mitad de las opciones ofrecidas.

Expone la idea con poca precisión.

Contesta de manera general.

\section{Acierto total: valor 1}

Contesta de manera completa lo que se le pregunta.

Claridad y precisión en su respuesta.

Coherencia en su respuesta.

Especifica las situaciones o ejemplos que se le solicitan.

Contesta correctamente ocho o más de 10 opciones.

Agrega algo más de lo que se le pide.

Es importante resaltar que por el tipo de estudio, aun cuando se aplican algunas herramientas de tipo cuantitativo, en el análisis de resultados predomina un enfoque de corte cualitativo porque básicamente se analizan las características de las respuestas brindadas a los ejercicios en cada una de las cinco categorías estudiadas.

\section{Confiabilidad de registro}

Se contactó con un psicólogo quien no tenía ningún antecedente sobre los objetivos de la investigación ni conocía las calificaciones asignadas por la investigadora; también se le entrenó sobre las opciones de calificación. Se eligieron 15 ejercicios al azar entre los 85 posibles para cotejar la calificación en las cinco variables estudiadas. Se compararon sus respuestas con las asignadas por la investigadora en los mismos ejercicios y se encontró un $82 \%$ de consistencia interjueces. 
Las etapas del análisis se llevaron a cabo de la siguiente manera: en un primer nivel de análisis se sintetizaron las respuestas y se anotaron en unas matrices impresas, separando los participantes con educación media superior y los profesionistas; en un segundo nivel se realizó el análisis interpretativo de los resultados para identificar las características de las respuestas brindadas por los participantes, en relación con patrones generales, expresión de juicios o valores, planteamiento de necesidades, dificultades en ciertos ejercicios, así como si realizaban ejercicios de tipo discriminativo, descriptivo, propositivo o reflexivo; en un tercer nivel se contrastó contra las teorías que sirvieron como base a la presente investigación, con el fin de confirmar o negar los postulados teóricos y conformar así el apartado correspondiente a la discusión.

La consideración del tipo de respuesta se realizó con base en los siguientes criterios:

Las respuestas de tipo discriminativo (d) son aquéllas donde los participantes requieren hacer operaciones de diferenciación como identificar cierto o falso a partir de una serie de propuestas; indicar diferencias entre conceptos, ideas, situaciones; relacionar columnas. También se da este valor cuando al solicitarse una operación de tipo reflexivo las personas exponen ideas generales, sin especificar una situación concreta, utilizando sólo una palabra como puede ser un verbo o un adjetivo en lugar de exponer razones o argumentos.

Las respuestas con carácter descriptivo (D) son aquéllas donde las personas refieren o relatan situaciones que viven a diario en su trabajo durante el ejercicio de sus funciones, en especial problemas en su área específica o entre diferentes áreas. Asimismo, cuando enumeran requisitos, cualidades o pasos de alguna situación; exponen ejemplos; relatan alguna experiencia; mencionan los pasos de un proceso; contestan a una serie de interrogantes directas, tipo qué, dónde, cuándo, cuánto. En ocasiones, de acuerdo con el ejercicio, deben realizar primero una operación discriminativa y luego una descriptiva, como en el caso de elegir en una lista de elementos que se les presenta y de ahí dar ejemplos; en este caso se evalúa la respuesta como parcial si sólo selecciona la opción, pero no realiza la segunda parte; mientras que la respuesta es completa si atiende las dos partes.

Las respuestas que se registran como propositivas (P) son aquéllas donde se pide que aporten nuevas modalidades a las situaciones que se les plantean como las siguientes: que planteen soluciones a problemas, formas de trabajo diferentes, mejores prácticas; que expliquen de qué manera puede llevarse a cabo algo; que 
planteen propuestas para aprender; que expongan un plan para la situación que se les plantea; que decidan las cosas que deben considerarse para atender alguna situación; que expresen sus metas de diferentes tipos. Puede haber ejercicios donde se requiere que realicen una operación discriminativa eligiendo entre una lista que se presenta y de ahí elaboren una proposición con base en su elección; en este caso se evalúa como respuesta parcial si sólo responde a la parte discriminativa y respuesta completa si realiza las dos partes.

Las respuestas de tipo reflexivo (R) son aquéllas donde las personas realizan una labor de análisis y con esta base llegan a determinadas conclusiones. En este sentido, se registran como respuestas reflexivas cuando las personas: identifican ideas principales; meditan y exponen su opinión; hacen una introspección sobre valores, hábitos o algún aspecto que se les plantee; meditan sobre sus necesidades personales en diferentes ámbitos; exponen sus conclusiones sobre algo; realizan una autoevaluación; hacen transferencias de técnicas o ideas que se presentan a situaciones reales; aportan una propuesta innovadora; analizan una situación de su entorno de trabajo o personal; determinan una jerarquía de tipo valorativo; hacen un diagnóstico o evaluación; realizan analogías; comentan sus sentimientos sobre situaciones de trabajo o personales vividas; exponen argumentos o razones sobre algo que se plantea; relacionan dos o más ideas o planteamientos para dar una respuesta o determinan consecuencias; identifican cosas en común; y establecen causas y efectos de un problema o situación.

\section{Principales resultados}

Entre los resultados encontrados, se muestra a continuación el comparativo de respuestas a los ejercicios donde se registró mayor dificultad en cada una de las cinco categorías por grupo; asimismo, se comparan las respuestas con dificultades de las sesiones dirigidas contra las autodirigidas con relación a la escolaridad.

Concentrado de resultados de ejercicios con dificultades por categoría y grupo

\begin{tabular}{|c|c|c|c|c|c|c|c|c|c|c|}
\hline Categoría & \multicolumn{2}{|c|}{ Análisis } & \multicolumn{2}{|c|}{ Relación } & \multicolumn{2}{|c|}{ Comparación } & \multicolumn{2}{|c|}{ Aplicación } & \multicolumn{2}{|c|}{ Deducción } \\
\hline & G-A & G-B & G-A & G-B & G-A & G-B & G-A & G-B & G-A & G-B \\
\hline $\begin{array}{l}\text { Respuesta } \\
\text { incompleta }\end{array}$ & 20 & 13 & 45 & 43 & 34 & 34 & 24 & 32 & 35 & 25 \\
\hline $\begin{array}{l}\text { Respuesta } \\
\text { en blanco }\end{array}$ & 7 & 11 & 7 & 6 & 10 & 10 & 11 & 11 & 6 & 13 \\
\hline $\begin{array}{l}\text { Respuesta } \\
\text { incorrecta }\end{array}$ & -- & 1 & 3 & 5 & 0 & 6 & 1 & 3 & 2 & 4 \\
\hline Total & 27 & 25 & 55 & 50 & 44 & 48 & 36 & 46 & 43 & 42 \\
\hline
\end{tabular}


A partir de estos resultados, el examen de las frecuencias de respuestas incompletas, en blanco e incorrectas, reveló que los ejercicios de la categoría Relación son los que presentan mayor índice de dificultad para ambos grupos de participantes.

En general, aparecieron una gran cantidad de respuestas parciales en los ejercicios de las diferentes categorías, aunque la dimensión Análisis registró el menor número. Con respecto al número de respuestas en blanco, ambos grupos obtuvieron la misma cantidad en las dimensiones comparación y aplicación; mientras que en las dimensiones Análisis y Deducción la frecuencia fue más elevada en el grupo B; por otra parte, el número de respuestas incorrectas fue menor en el grupo A que en el $\mathrm{B}$.

Para comparar los resultados de las sesiones dirigidas contra las autodirigidas de los participantes de educación media contra los ingenieros, se procedió a determinar las frecuencias de las respuestas en blanco, las incorrectas y las incompletas para identificar en cuáles sesiones ocurrieron este tipo de situaciones. Los resultados se pueden observar en los siguientes cuadros, con los datos en negritas denotando los totales.

Comparación de respuestas con dificultades por tipo de sesión, categoría y escolaridad

\begin{tabular}{|c|c|c|c|c|c|c|c|c|c|c|c|c|c|c|c|c|c|c|}
\hline \multicolumn{19}{|c|}{ CATEGORÍA ANÁLISIS } \\
\hline \multicolumn{10}{|c|}{ Sesiones dirigidas } & \multicolumn{9}{|c|}{ Sesiones autodirigidas } \\
\hline \multicolumn{5}{|c|}{ Educ. Media } & \multicolumn{4}{|c|}{ Profesionistas } & & \multicolumn{5}{|c|}{ Educ. Media } & \multicolumn{4}{|c|}{ Profesionistas } \\
\hline Resp & 1 & 2 & 4 & 5 & Total & 1 & 24 & 5 & Total & 3 & 6 & 7 & 8 & Total & 3 & 6 & 8 & Total \\
\hline RI & 1 & & & & 1 & & & & -- & & & & & -- & & & & -- \\
\hline $\mathrm{NC}$ & 3 & & 1 & & 4 & 2 & 2 & & 4 & 2 & 1 & 1 & 2 & 6 & 3 & 1 & 3 & 7 \\
\hline $\mathrm{RP}$ & 3 & & & & 3 & 3 & 4 & & 7 & 2 & 2 & & 4 & 8 & 2 & 1 & 27 & 12 \\
\hline $\begin{array}{l}\text { Subtotal } \\
\text { sesión }\end{array}$ & 7 & & 1 & & & 5 & 6 & & & 4 & 3 & 1 & 6 & & 5 & 2 & 210 & \\
\hline
\end{tabular}

Los resultados anteriores muestran que en la categoría Análisis aparecieron más respuestas en blanco e incompletas en las sesiones autodirigidas, que en las dirigidas en ambos grupos. 


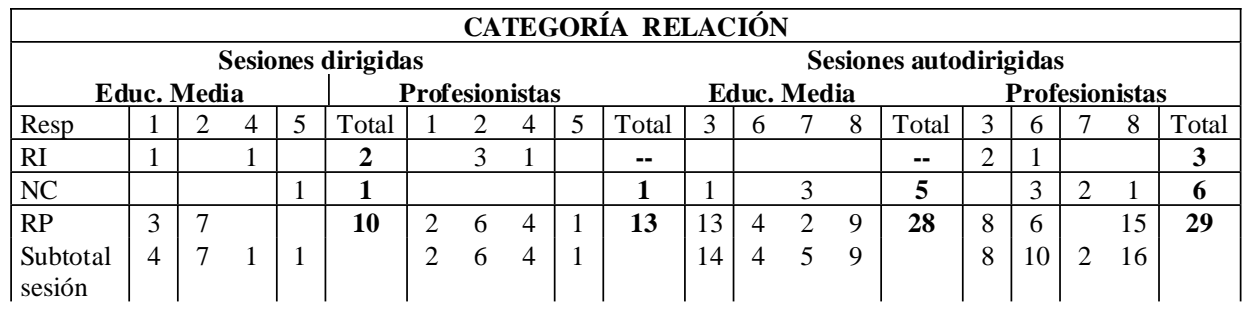

Como se observa en el cuadro, la categoría Relación registró un elevado número de respuestas incompletas en las sesiones autodirigidas con respecto a las sesiones dirigidas por parte de ambos grupos.

\begin{tabular}{|c|c|c|c|c|c|c|c|c|c|c|c|c|c|c|c|c|c|c|c|c|}
\hline \multicolumn{21}{|c|}{ CATEGORÍA COMPARACIÓN } \\
\hline \multicolumn{10}{|c|}{ Sesiones dirigidas } & \multicolumn{11}{|c|}{ Sesiones autodirigidas } \\
\hline \multicolumn{5}{|c|}{ Educ. Media } & \multicolumn{5}{|c|}{ Profesionistas } & \multicolumn{6}{|c|}{ Educ. Media } & \multicolumn{5}{|c|}{ Profesionistas } \\
\hline Resp & 1 & 2 & 4 & 5 & Total & 1 & 2 & 4 & 5 & Total & 3 & 6 & 7 & 8 & Total & 3 & 6 & 7 & 8 & Total \\
\hline RI & & 2 & 1 & 1 & 4 & & & & & - & & 2 & 1 & 2 & 5 & & & & & -- \\
\hline $\mathrm{NC}$ & & 1 & 1 & 4 & 6 & & 1 & 1 & 2 & 4 & & & 4 & & 4 & 2 & 1 & 1 & & 4 \\
\hline $\mathrm{RP}$ & & 4 & 6 & 4 & 14 & & 2 & 2 & 4 & 8 & 6 & 1 & 10 & & 17 & 7 & & 12 & 1 & 20 \\
\hline $\begin{array}{l}\text { Total } \\
\text { sesión }\end{array}$ & & 7 & 8 & 9 & & & 3 & 3 & 6 & & 6 & 3 & 11 & & & 9 & 1 & 13 & 1 & \\
\hline
\end{tabular}

En la categoría Comparación se registró también un número importante de respuestas incompletas, aunque menor que en la categoría anterior. Esta situación se apreció en mayor medida en el grupo de profesionistas que en el grupo de educación media, inclusive en este último grupo el número de respuestas incompletas de las sesiones autodirigidas tuvo poca diferencia en relación con las dirigidas.

\begin{tabular}{|c|c|c|c|c|c|c|c|c|c|c|c|c|c|c|c|c|c|c|c|c|}
\hline \multicolumn{21}{|c|}{ CATEGORÍA APLICACIÓN } \\
\hline \multicolumn{11}{|c|}{ Sesiones dirigidas } & \multicolumn{10}{|c|}{ Sesiones autodirigidas } \\
\hline \multicolumn{5}{|c|}{ Educ. Media } & \multicolumn{5}{|c|}{ Profesionistas } & \multicolumn{6}{|c|}{ Educ. Media } & \multicolumn{5}{|c|}{ Profesionistas } \\
\hline Resp & 1 & 2 & 4 & 5 & Total & 1 & 2 & 4 & 5 & Total & 3 & 6 & 7 & 8 & Total & 3 & 6 & 7 & 8 & Total \\
\hline RI & & & 1 & & 1 & & & & & - & & & & & - & & & & 1 & 1 \\
\hline $\mathrm{NC}$ & 1 & & 2 & & 3 & 1 & 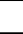 & & & 2 & 1 & 1 & 3 & 5 & 10 & 2 & 3 & & 3 & 8 \\
\hline $\mathrm{RP}$ & 2 & 8 & 1 & 5 & 16 & 2 & 2 & 1 & 4 & 9 & 2 & 3 & 5 & 7 & 17 & & 6 & 1 & 4 & 11 \\
\hline $\begin{array}{l}\text { Subtotal } \\
\text { sesión }\end{array}$ & 3 & 8 & 4 & 5 & & 3 & 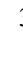 & 1 & 4 & & 3 & 3 & 8 & 12 & & 2 & 9 & & 8 & \\
\hline
\end{tabular}

En la categoría Aplicación el número de respuestas incompletas fue muy parecido en las sesiones dirigidas y en las autodirigidas; sin embargo, las respuestas en blanco de ambos grupos registraron una mayor frecuencia en las sesiones autodirigidas que en las dirigidas. 


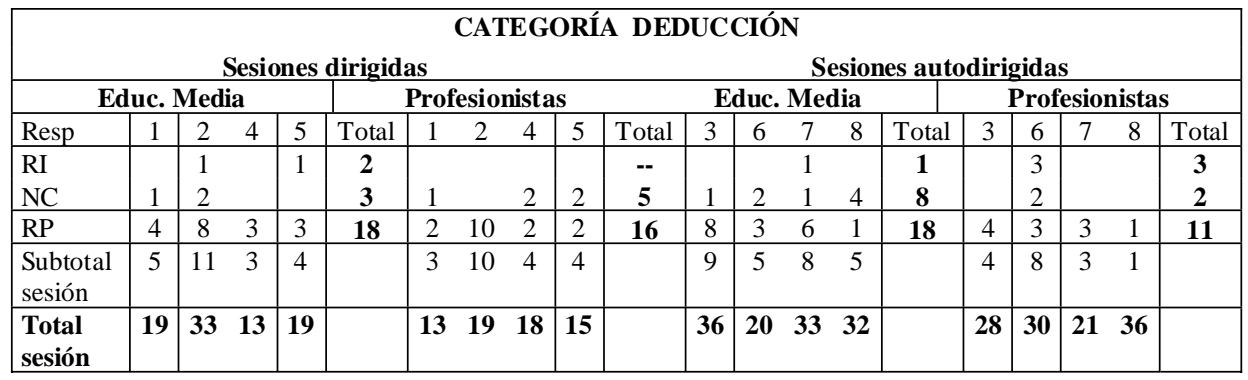

En la categoría Deducción, el número de respuestas incompletas en el grupo con educación media fue muy similar en las sesiones dirigidas que en las autodirigidas; no obstante, aparecieron ocho respuestas en blanco en las sesiones autodirigidas contra tres en las dirigidas. El grupo de profesionistas registró 16 respuestas incompletas en las sesiones dirigidas contra 11 de las autodirigidas; asimismo, aparecieron dos respuestas en blanco en las sesiones autodirigidas contra cinco en las dirigidas.

De los resultados generales por sesión se encontró que, de las sesiones autodirigidas, la sesión tres, referente al análisis de la ejecución, pareció resultar especialmente difícil para los participantes con educación media, ya que registraron un total de 36 respuestas con diferentes dificultades contra 28 del grupo de profesionistas. En la sesión seis los participantes con licenciatura registraron 30 respuestas de este tipo contra 20 del grupo con educación media. En la sesión siete los participantes del grupo de educación media presentaron 33 respuestas con diferentes dificultades contra 21 del grupo de profesionistas. En la sesión ocho se registraron 32 respuestas de este tipo en el grupo de educación media contra 36 del grupo de profesionistas. En las sesiones dirigidas, se registraron 33 respuestas con diversas dificultades en el grupo de educación media, contra 19 de los profesionistas.

La reflexión sobre la elevada frecuencia de ejercicios donde se registraron respuestas incompletas plantea la necesidad de hacer una evaluación más profunda de los mismos, así como de retroalimentarlo con los participantes para identificar si estos resultados se dieron por falta de tiempo, falta de claridad en la redacción o bien por desinterés en responderlos. Asimismo, convendría realizar un análisis más profundo considerando el tipo de ingeniería con el fin de detectar características más finas en las respuestas.

Otros resultados se refieren al tipo de necesidades de aprendizaje dentro de la empresa, relacionadas en general con los participantes en lo referente a lo que les 
afecta directamente en la realización de sus funciones y la consecución de las metas que tienen fijadas. Se pudo apreciar que otorgan una connotación muy amplia a las necesidades de aprendizaje no sólo como forma de mejorar las funciones directas de su puesto de trabajo, sino para señalar requerimientos de otras personas, áreas o autoridades y para referirse a aspectos de tipo organizacional como pueden ser canales de comunicación, programas que desarrollan en la empresa, pero sin especificar con claridad qué tipo de aprendizaje requieren. En este sentido, pasan del aprendizaje individual al plano del aprendizaje organizacional.

Con respecto a las modalidades que proponen para instrumentar el aprendizaje entre áreas, los resultados brindados por los 24 ingenieros y los 13 participantes con educación media se pueden apreciar en el siguiente cuadro:

\section{Modalidades para instrumentar el aprendizaje entre áreas}

\begin{tabular}{l|c|c|}
\hline \multicolumn{1}{|c|}{ Modalidad } & Educación media & Profesionistas \\
\hline Plan integral de capacitación & 6 & 7 \\
\hline Reuniones para solucionar problemas & 10 & 11 \\
\hline Formación de coachings & 3 & 3 \\
\hline Realización de proyectos conjuntos & 2 & 9 \\
\hline Lecturas de reflexión & - & 2 \\
\hline Mentores técnicos & - & - \\
\hline Formación de equipos especializados & 2 & 2 \\
\hline Diplomados o maestrías & - & 4 \\
\hline Cursos técnicos especializados & 2 & 7 \\
\hline Programas de autoaprendizaje & - & 1 \\
\hline Oportunidades de aprendizaje informal & - & 1 \\
\hline Fomento a la participación a todos niveles & 2 & 3 \\
\hline Compartir información y conocimientos & 5 & 8 \\
\hline Aprender haciendo & - & 7 \\
\hline Identificación y discusión de mejores prácticas & 3 & 5 \\
\hline
\end{tabular}

Estos resultados sugieren que el aprendizaje en la empresa lo vinculan ambos grupos (educación media y profesionistas) con la solución de problemas cotidianos, con la posibilidad de compartir información y conocimientos y con la oportunidad de contar con un programa de capacitación formal. Las situaciones de carácter informal o de autoaprendizaje no son muy señaladas por los participantes de ambos grupos. Por otro lado, también se puede apreciar preferencias por parte de 
los profesionistas por participar en proyectos entre áreas, así como aprender haciendo, lo que sugiere una posible necesidad de experimentar para hacer mejoras.

En relación con la orientación para mejorar el desempeño, algunas propuestas se encaminan claramente al aprendizaje de valores, en tanto que otras se enfocan hacia aspectos de carácter técnico, especialmente ligadas a cuestiones de mantenimiento, problemas con materiales y falta de información, así como de tipo administrativo como es la necesidad de aprender a comunicarse y a seguir procedimientos. Sin embargo, las cuestiones relacionadas con logística, así como con aspectos de tipo organizativo propios de la empresa, sugieren la necesidad de aprendizaje en el nivel organizacional para corregir anomalías percibidas por algunos participantes, como la deficiencia en la estructura, la inexistente planeación, así como el agobio y confusión en el trabajo cotidiano.

\section{Conclusiones}

La importancia de considerar las habilidades del pensamiento en el ámbito laboral es innegable, puesto que fortalece a los individuos a partir de sus propios potenciales; asimismo, se puede dar un resultado favorable en el nivel grupal a partir del efecto sinérgico resultante de conjuntar las diferentes capacidades.

Las dificultades de estudiar las habilidades del pensamiento resultan evidentes; por ello, la presente investigación sólo realiza contribuciones modestas a este tópico. El entrenamiento logra que las personas valoren la importancia de aprender de manera permanente dentro de su empresa, así como, en muchos casos, a través de planes de educación formal; esto se comprobó a través de las respuestas que brindaron en los diferentes ejercicios que particularmente hacen referencia sobre estos aspectos. En especial, se considera importante para investigaciones posteriores profundizar en una o dos habilidades con el objeto de hallar elementos más concluyentes.

En general, se identifica un patrón de respuestas de tipo práctico en las respuestas a las cinco categorías de pensamiento; asimismo, se aprecian mejores resultados en las sesiones dirigidas para las categorías Análisis, Relación y Comparación; por su parte, resultados muy similares entre las sesiones dirigidas y autodirigidas en la categoría Aplicación; por lo que respecta a la categoría Deducción se registran resultados iguales en ambos tipos de sesiones en el grupo de educación media, en cambio los profesionistas obtienen mejores resultados en las sesiones dirigidas. 
Se observó que las respuestas en blanco registran una mayor frecuencia en las sesiones autodirigidas que en las dirigidas en los dos grupos, educación media y profesionistas, lo cual sugiere que la conducción por parte de la investigadora facilita en cierta medida la obtención de resultados favorables.

En términos generales, dentro de la categoría Análisis se presentaron inconsistencias en el esfuerzo por reflexionar con los participantes de ambos grupos; esto puede deberse al menor o mayor interés por ciertos ejercicios, por lo que se deriva la necesidad de revisar de manera pormenorizada cada uno de estos ejercicios a fin de rediseñarlos.

Por otra parte, se registró un equilibrio entre el aprendizaje enfocado en cuestiones humanas, en aspectos técnicos y de carácter administrativo. La importancia de aprender en el lugar de trabajo la asocian, en general, con fines de beneficio personal, ya sea por resolver necesidades inmediatas tanto para mantener su empleo como para conseguir resultados y obtener ascensos. Una minoría menciona aspectos de crecimiento y satisfacción personal. En este sentido influyen las situaciones de presión cotidiana que experimentan las personas y que limitan las posibilidades de dar un espacio al aprendizaje de carácter más reflexivo.

En general se aprecia cierta resistencia por reflexionar sobre cuestiones personales, aunque esto es más notorio en el grupo de educación media; sin embargo, ambos grupos tienden a identificar claramente las deficiencias que observan en el desempeño global de la empresa, aunque sin considerar su propia participación para resolverlos.

Es importante destacar el alto nivel de homogeneidad en las respuestas de los participantes con educación media, en comparación con los profesionistas, en aspectos de tipo práctico relacionados con el desempeño cotidiano, con excepción de algunos ejercicios donde se encontró que la escolaridad sí influye para la correcta ejecución de los mismos. La misma situación se registró en cuanto a la comprensión de los contenidos de algunas sesiones, en particular la segunda, que representó dificultades para el grupo con educación media. Estos resultados dejan abierta la interrogante sobre las posibles diferencias debidas a la escolaridad al considerar ejercicios que no sólo se circunscriban al ámbito de trabajo.

Los participantes de ambos grupos establecen con precisión un diagnóstico de las problemáticas que detectan en su trabajo; no obstante, la gran mayoría no conside- 
ra su propia contribución en éstas. En este sentido, las personas con escolaridad media dan respuestas muy similares a las brindadas por los ingenieros, tanto en aspectos técnicos como de relaciones interpersonales, particularmente entre áreas.

En la categoría Relación, todos los participantes en general transfieren con facilidad el vocabulario del pensamiento a situaciones de su trabajo cotidiano; asimismo, establecen relaciones causales de procesos, tiempos y personas de las diferentes situaciones problemáticas que se les plantean e identifican condiciones para que se den resultados en su trabajo.

Se aprecia sensibilidad por los participantes de ambos grupos con respecto a los valores, especialmente los vinculados con las relaciones entre las personas de diferentes áreas. Los participantes de educación media parecen necesitar respeto, trato igualitario y apoyo; en tanto que los ingenieros parecen requerir reconocimiento y trabajo en equipo.

Se detectan características en la cultura empresarial que bloquean el aprendizaje, especialmente la falta de planeación, que lleva a una constante falta de claridad en cuanto a los objetivos y resultados que se espera de las personas.

En la categoría Comparación, los participantes de ambos grupos establecen diferencias y semejanzas entre ideas y situaciones planteadas; además, determinan jerarquías cuantitativas y cualitativas para aspectos de desempeño, como en cuestiones relacionadas con valores. En esta categoría se requiere diseñar ejercicios que detecten aspectos más finos al comparar y diferenciar elementos constituyentes de situaciones o problemas que viven en su práctica cotidiana.

En la especificación de metas se observa mayor precisión por parte del grupo de ingenieros con respecto al grupo con educación media, que plantea cuestiones más generales. También con relación al enfoque a resultados, las personas con educación media exhiben resultados más vagos en comparación con los ingenieros que plantean ideas más concisas. En este sentido la escolaridad influye especialmente en el manejo del lenguaje, así como en la posibilidad de circunscribir situaciones.

En lo referente a la categoría Aplicación, tanto los participantes con educación media como los profesionistas transfieren algunas técnicas revisadas a las situaciones planteadas, con excepción de ciertas herramientas para pensar en las que se detectaron dificultades para responderlas. 
Las posibilidades de innovación se aprecian de manera similar en los participantes con educación media, así como en los profesionistas, pues en general se registraron respuestas semejantes en cantidad y calidad en los ejercicios relacionados con este tema; sin embargo, también en ambos grupos se detectan algunos participantes que no realizan mucho esfuerzo por hacer propuestas innovadoras. Estos resultados pueden deberse tanto a cualidades personales como también a cuestiones culturales de la empresa que limitan o favorecen la innovación.

Por lo que respecta a la intervención de los participantes para resolver problemas que les dificultan alcanzar sus metas, se registró una posición más pasiva en los individuos con educación media en relación con los profesionistas, quienes adoptan un enfoque más participativo.

El instrumento de recolección de datos permite realizar un diagnóstico de necesidades de aprendizaje encaminadas a mejorar el desempeño en el trabajo. Con relación al diagnóstico de los problemas de su entorno laboral, ambos grupos presentan un panorama claro y detallado porque en general exponen con facilidad los ejemplos que ilustran situaciones de diferentes tipos relacionados con su trabajo; asimismo, aplican diferentes herramientas para analizar las situaciones circunscritas en su área de trabajo.

Se puede apreciar que en la incorporación de las habilidades del pensamiento para aprender en su trabajo, los participantes de ambos grupos le asignan una connotación muy amplia, puesto que la consideran una herramienta para mejorar en su trabajo, en la solución de problemas, así como en las relaciones interpersonales.

En la variable Deducción se registran algunos ejercicios que fueron resueltos con facilidad por los participantes de ambos grupos; sin embargo, en otros se presentaron dificultades, como es el caso de la especificación de su proceso de aprendizaje en alguna situación cualquiera, porque sólo aportan generalidades, sin considerar las dificultades vividas, sentimientos despertados, así como las satisfacciones experimentadas. Estos resultados conducen a que se considere si las instrucciones son suficientemente claras, si se requiere especificarlas con mayor precisión, o bien revisar si la extensión del ejercicio genera cierta confusión.

Se observó que las cuestiones que requerían vincular ideas para derivar inferencias tuvieron un mayor grado de dificultad para ser resueltas por los participantes de ambos grupos, como es el caso de la relación entre metas y desempeño; sin em- 
bargo, otros planteamientos de este tipo sí los responden con facilidad como es el caso de encontrar los aspectos en común en los catalizadores de cambios.

Los participantes de los grupos educación media y profesionistas enfocan con facilidad el aprendizaje en la empresa a su ámbito de trabajo inmediato y en menor medida hacia otras áreas. Esto plantea la necesidad de que el aprendizaje sea resultado de un cambio en la cultura donde se le considere como parte integral del trabajo, donde se involucre a todas las áreas, así como al personal de diferentes niveles jerárquicos.

Los participantes con educación media presentan más dificultades para identificar sus necesidades de aprendizaje, en función de su desempeño, en comparación con los ingenieros. Esto puede sugerir que el nivel de mecanización, la rutina y las presiones cotidianas dificultan ampliar las perspectivas de aprendizaje en las personas con menor escolaridad; sin embargo, se requieren estudios adicionales para comprobar estas ideas.

En general, los participantes de ambos grupos muestran motivación personal hacia su trabajo, aun con el ambiente de incertidumbre y problemas que viven a diario. Esto se comprueba por los comentarios favorables que dan de su empresa.

Los supervisores y los gerentes aceptan como responsabilidad importante dentro de sus funciones como administradores liberar el potencial de las personas a su cargo para llevar el pensamiento en acción como estrategia para enfrentar la complejidad de la vida cotidiana en el trabajo.

Dentro de las empresas, mantener la capacidad de indagación del personal en todos los niveles se convierte en un elemento fundamental, puesto que tiene que ver, entre otras, con mantener la curiosidad, así como fomentar el cuestionamiento sobre los problemas que suceden en el día a día. En este sentido, el fortalecimiento de las habilidades de pensamiento a nivel individual puede servir como estrategia de competitividad para las empresas.

Por otra parte, la gestión del conocimiento debe buscar, entre otras, reducir al máximo los bloqueos organizativos que inhiben el aprendizaje, fomentar la innovación, evitar la rutina, mantener una comunicación abierta, favorecer la iniciativa, así como el compromiso por los resultados conjuntos, fortalecer el liderazgo del personal con mando a todos niveles, mantener un elevado espíritu de equipo elimi- 
nando las barreras, entre áreas, a partir de la creación de una nueva cultura como organización enfocada al conocimiento.

Es necesario profundizar en la validez de los ejercicios diseñados para medir cada una de las categorías del pensamiento estudiadas, sobre todo en cuanto a los rangos de dificultad a lo largo del entrenamiento. De igual forma, es importante realizar esta investigación con grupos de otras profesiones, así como en empresas de diferentes giros, con el propósito de comparar los resultados obtenidos. Asimismo, se requiere hacer adaptaciones al instrumento para aplicarlo a personas de menor escolaridad.

En general, se obtuvieron resultados favorables en cuanto a la posibilidad de fomentar las habilidades del pensamiento como herramientas de aprendizaje; sin embargo se necesita profundizar más en cada una de ellas, así como trabajar con mucho más detalle sus operaciones cognoscitivas.

Indudablemente, resulta importante reforzar este tipo de investigación con el seguimiento en el desempeño del trabajo con el fin de verificar si realmente se aplican las estrategias de pensamiento practicadas durante el entrenamiento, en su trabajo cotidiano.

La presente investigación tiene limitaciones en cuanto al número de personas que participaron, lo que impide la generalización de los resultados encontrados. La restricción de tiempo fue también un factor que pudo influir en los resultados, en particular de las personas con menor escolaridad.

Es fundamental incluir tiempo de retroalimentación como parte del entrenamiento para identificar las causas de los errores registrados en los diferentes ejercicios, así como verificar la comprensión de contenidos, precisión del lenguaje y aplicación de las diversas herramientas.

Finalmente, es conveniente continuar en esta línea de investigación que permita contribuir en mayor medida la relación del conocimiento, el aprendizaje y el pensamiento en las organizaciones. 


\section{Referencias bibliográficas}

Argyris, C. y D. Schon (1990), Organizational Learning: a theory of action perspective. Reading, Addison-Wesley, San Francisco, California.

Ausubel, D., Novak J. y H. Hanesian (1973), Psicología educativa. Un punto de vista cognoscitivo, Trillas, México.

Bereiter, C. y M. Scardamalia (1985), "Cognitive coping strategies and the problem of inert-knowledge”, en S. Chipman, Segal J. y R. Glaser (Comps.), Thinking and learning skills. Research and open questions, vol. 2, Lawrence Erlbaum Associates, Hillsdale, Nueva Jersey.

Boud, D. y J. Garrick (1999), Understanding learning at work, Londres, Routledge.

Candy, P. y J. Matthews (1998), en Understanding learning at work, Londres, Routledge.

Derry, S. y D. Murphy (1986), "Designing systems that train learning ability: From theory to practice", en Review of Educational Research, 56, pp. 1-39.

Gore, E. (1998), La educación en la empresa, Granica, Barcelona.

Labarrere, Arthur (1994), Pensamiento, análisis y autorregulación en la actividad cognoscitiva de los alumnos, Ángeles Editores, México.

Marzano, Robert et al. (1988), Dimensions of thinking. A framework for curriculum and instruction, Association for Supervision and Curriculum Development (ASCD), Alexandria, Virginia.

Presseisen, G., en Costa A. (2001), Developing Minds, Association for Supervision and Curriculo Development, Virginia.

Schein, E. (1999), "On dialogue, culture, and organizational learning", en Organizational Dynamics, Vol. 22 (2), otoño, pp. 124-135. 